Manuelle Medizin 2019 · 57:439-440

https://doi.org/10.1007/s00337-019-00610-3

(c) Springer Medizin Verlag GmbH, ein Teil von Springer Nature 2019

Soeben ist in Deutsches Ärzteblatt die S3Leitlinie „Funktionelle Körperbeschwerden“ erschienen [1, 2]. Diese Leitlinie zeigt deutlich die Mängel der modernen apparativ orientierten Medizin auf: Was in der Bildgebung oder in paraklinischen Untersuchungsmethoden (z. B. Labor, EKG, EMG) nicht nachweisbar ist, muss einer psychosomatischen Diagnostik und Therapie zugeführt werden.

Schon vor 3 Jahren erkannte die Arbeitsgruppe Funktionskrankheiten der Ärztevereinigung für Manuelle Medizin, in enger Verbindung mit der inzwischen in der klinischen Medizin fest etablierten Schmerzerkrankung, pathologisch strukturell nicht fassbare Funktionsstörungen des Stütz- und Bewegungssystems, die nur einer fein graduierten manualmedizinischen Untersuchung zugänglich sind.

Von der (normalen) Funktion als Aufgabe eines Organs, eines Gewebes oder einer Zelle kommt es durch viele Kontextfaktoren $\mathrm{zu}$ Funktionsstörungen, die zeitlich unterschiedlich lang kompensiert werden und für den betroffenen Patienten unerkannt bleiben. Die Ursachen können vielfältig sein, von muskulären Dysbalancen durch Einschränkungen der konditionellen Fähigkeiten der Muskulatur und damit einer Diskrepanz zwischen Belastung und Belastbarkeit bis hin zu Veränderungen der muskulären Stereotypien der Gelenkführung. Bestehen diese Funktionsstörungen über eine längere Zeit oder kommen neue Anforderungen hinsichtlich Belastung hinzu, kann dies zur Ausprägung von Funktionskrankheiten führen. Hauptsächlich wird der Schmerz

E. Seidel ${ }^{1} \cdot$ L. Beyer ${ }^{2}$

'Sophien- und Hufelandklinikum Weimar, Weimar, Deutschland

${ }^{2}$ Ärztehaus Mitte, Jena, Deutschland

\title{
Funktionskrankheiten - Einführung zu einem Denk- und Handlungskonzept
}

den Patienten in die Sprechstunde führen, aber auch zunehmende Funktionsbehinderungen im Alltag und Beruf. Dies müssen und können nicht immer sofort messbare Funktionseinschränkungen sein, so kann z.B. nur die Ausdaueroder die Koordinationsleistung eingeschränkt sein. Typische Merkmale einer strukturellen Erkrankung fehlen (Labor, Röntgen, MRT, EMG), somit erfolgt nach einer typischen apparativ geprägten klinischen Diagnostik sehr schnell die Zuordnung einer psychosomatischen Funktionserkrankung (s. Leitlinie).

Eine kausale Therapie bzw. Intervention in den Entstehungsmechanismus einer gestörten Funktion ist in diesem Falle nicht mehr gegeben.

Somit ist immer vor der Einleitung therapeutischer Schritte zwingend eine manualmedizinische Untersuchung hinsichtlich vorhandener Funktionsstörungen und -erkrankungen notwendig. Dies setzt jedoch Kenntnisse des multifaktoriellen Bedingungsgefüges voraus, die in den nachfolgenden Thesen ausführlich dargestellt werden.

\section{》) Vor Einleitung the- rapeutischer Schritte ist eine manualmedizinische Untersuchung notwendig}

Erst wenn die Untersuchung abgeschlossen ist, können und sollten bei Bedarf die weiteren diagnostischen und therapeutischen Schritte der psychosomatischen Differenzialdiagnostik und -therapie folgen.
Von großer Bedeutung sind die nachfolgenden Thesen für alle Fragen des betrieblichen Gesundheitsmanagements und der Präventionsansätze aller Kostenträger im Gesundheitssystem (Berufsgenossenschaft, gesetzliche Krankenversicherung, Deutsche Rentenversicherung).

Beispielsweise führen einseitige $\mathrm{Be}$ lastungsabläufe (Bildschirmarbeitsplatz, Fließbandarbeit) fast immer zur Entstehung von muskulären Dysbalancen und nachfolgend zu Störungen der Funktion.

Daraus können sich sekundär weitere, auch lokal getrennte Folgeprozesse ergeben. So ist beim Bildschirmarbeitsplatz die Entstehung der muskulären Dysbalance der Blickfolgemuskulatur und die damit verbundenen Veränderungen im Stereotyp vom „headmover“ zum „eyemover“ gut untersucht. Die muskuläre Problematik bleibt aber oft unentdeckt, die vom Patienten geschilderten für ihn primären Symptome (Sehstörungen, Kopfschmerzen) werden vorrangig diagnostiziert und therapiert. Die zugrunde liegende Funktionsstörung und -erkrankung der veränderten Funktionsweise der oberen Halswirbelsäule und des Schultergürtels werden nicht ausreichend berücksichtigt und die angefertigte Bildgebung ergibt keine Hinweise. So wird eine kausale Behandlung verhindert.

Das Beispiel Bildschirmarbeitsplatz zeigt eindrucksvoll, das funktionelles Denken v.a. einen präventiven Charakter hat. Es dient der Vermeidung von Funktionsstörungen und der rechtzeitigen Diagnostik von Funktionserkrankungen. 
Aus diesem Grunde halten wir es für zwingend notwendig, schon die Ausbildung von Ärzten und Therapeuten in diese Richtung wesentlich zu verbessern, die Präventionskonzepte unter der Beachtung der nachfolgenden Thesen $\mathrm{zu}$ überdenken und danach als festen Bestandteil u. a. in das betriebliche Gesundheitsmanagement $\mathrm{zu}$ übernehmen.

Erst dann wird es gelingen, strukturell-pathologisch nicht fassbare Beschwerdekomplexe der Patienten nicht immer nur der psychosomatischen $\mathrm{Me}$ dizin ohne den hierfür erforderlichen kausalen Ansatz zur Intervention zu überlassen. Ein interdisziplinärer Ansatz in einem multiprofessionellen Team ist hierfür unumgänglich, mit der erforderlichen Offenheit gegenüber allen teilnehmenden Professionen. Dann können wir für unsere Patienten einen optimalen Lösungsansatz gemeinsam auswählen.

Darüber hinaus sind aber präventive Strategien zur Vermeidung bzw. Reduktion von Funktionserkrankung weiterzuentwickeln und fest zu implementieren. Die Aufklärung des Patienten über das multifaktorielle Bedingungsgefüge wird dabei ein sehr wichtiger Bestandteil der therapeutischen Intervention sein. Wenn der Patient diese Zusammenhänge versteht und in sein künftiges Handeln einbezieht, wird eine erfolgreiche Therapie von Funktionserkrankungen gelingen.

Auch hier gibt es sehr große Parallelen zur Schmerzerkrankung. Die Funktionskrankheit des Stütz- und Bewegungssystem sollte in Zukunft den gleichen Stellenwert erlangen, gerade weil die Implementierung dieses die Funktion und die Funktionsstörung aufgreifenden Gedankenguts in der Lage wäre, chronische Schmerzerkrankungen zu vermeiden. Gleichzeitig würde sie es ermöglichen, viele psychosomatisch stigmatisierte Patienten wieder einem kausalen, interdisziplinären Therapieansatz zuzuführen.

\section{Korrespondenzadresse}

Prof. Dr. E. Seidel

Sophien- und Hufelandklinikum Weimar

H.-v.-d.-Velde-Str. 2, 99425 Weimar,

Deutschland

e.seidel@klinikum-weimar.de
Interessenkonflikt. E. Seidel und L. Beyer geben an dass kein Interessenkonflikt besteht.

\section{Literatur}

1. Arbeitsgemeinschaft der Wissenschaftlichen Medizinischen Fachgesellschaften e.V. (2019) S3-Leitlinie "Funktionelle Körperbeschwerden". www.awmf.org/leitlinien/detail/ll/051-001.html. Zugegriffen:4. Sept. 2019

2. Roenneberg C, Sattel H, Schaefert R, Henningsen $P$, Hausteiner-Wiehle C (2019) Clinical practice guideline: functional somatic symptoms. Dtsch Arztebl Int 116:553-560. https://doi.org/10.3238/ arztebl.2019.0553
Auch online Zugang zu allen Beiträgen Ihres Zeitschriftenabonnements

Wussten Sie, dass Sie als Abonnent dieser Zeitschrift automatisch Online-Zugriffsrechte auf das gesamte Beitragsarchiv und die CME-Kurse haben?

Der Zugang zu Ihrer Online-Bibliothek und den CME-Kursen ist im Abonnement Ihrer Zeitschrift inbegriffen. Für den Zugang müssen Sie sich lediglich einmal über www.springermedizin.de/register registrieren.

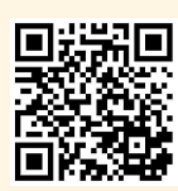

Über diesen QR-Code schnell und einfach registrieren

Bitte nutzen Sie für die Registrierung Vorund Nachname und Lieferadresse wie beim Abonnement der Zeitschrift (s. Adressaufkleber auf Ihrem Heft). So kann im System die Zugehörigkeit zu Ihrer Zeitschrift sichergestellt werden.

Aufgrund des Heilmittelwerbegesetzes dürfen die Inhalte der Website nur medizinischen Fachkreisen zur Verfügung gestellt werden. Bei der Anmeldung bitten wir Sie deshalb, einen Berufsnachweis vorzulegen.

Bei Medizinern mit Mitgliedschaft in der deutschen Ärztekammer reicht die einheitliche Fortbildungsnummer

(EFN). Alternativ schicken Sie eine Bestätigung des Arbeitgebers, Studiennachweis oder andere Zeugnisse ganz unkompliziert an kundenservice@springermedizin.de.

Mit Benutzername und Passwort haben Sie außerdem Zugang zu den freien Inhalten auf den Seiten von: www.springermedizin.de www.aerztezeitung.de

Sollten Fragen oder Probleme auftauchen, wenden Sie sich einfach an Ihren Kundenservice:

kundenservice@springermedizin.de 